\title{
EGFR Mutations in Cell-free Plasma DNA from Patients with Advanced Lung Adenocarcinoma: Improved Detection by Droplet Digital PCR
}

\author{
Anna Buder ${ }^{1}$. Ulrike Setinek ${ }^{2} \cdot$ Maximilian J. Hochmair $^{3} \cdot$ Sophia Schwab $^{3} \cdot$ Klaus Kirchbacher $^{4} \cdot$ Andrea Keck $^{4}$. \\ Otto C. Burghuber ${ }^{3} \cdot$ Robert Pirker $^{5} \cdot$ Martin Filipits $^{1}$
}

Published online: 27 February 2019

(c) The Author(s) 2019

\begin{abstract}
Background Analysis of cell-free DNA from blood could provide an alternative method for identifying genomic changes in the tumors of patients with advanced lung adenocarcinoma.

Objective We compared the performance of droplet digital PCR (ddPCR) and Cobas ${ }^{\circledR}$ EGFR Mutation Test v2 (Cobas) for detecting EGFR mutations in cell-free plasma DNA.

Patients and Methods Plasma samples from patients with advanced $E G F R$-mutated lung adenocarcinoma were analyzed for EGFR T790M, exon 19 deletions, and L858R mutations by both ddPCR and Cobas.

Results T790M testing was performed in 354 plasma samples collected from 129 patients. The concordance rate between ddPCR and Cobas for T790M, sensitivity, and specificity were 86, 100, and 85\%, respectively. Exon 19 deletions were analyzed in 196 plasma samples obtained from 71 of the 129 patients using both platforms. The concordance rate between ddPCR and Cobas for exon 19 deletions, sensitivity, and specificity were 90, 92, and 89\%, respectively. L858R mutations were studied in 124 plasma samples obtained from 44 of the 129 patients using both assays. The concordance rate between ddPCR and Cobas for L858R, sensitivity, and specificity were 90, 91, and 89\%, respectively. In patients who progressed under treatment with an EGFR TKI $(n=50)$, the T790M positivity rate was $66 \%$ using ddPCR, but only $24 \%$ using Cobas. Conclusions We observed a high concordance between ddPCR and Cobas in detecting EGFR mutations in plasma samples of patients with advanced $E G F R$-mutated lung adenocarcinoma, but ddPCR was more sensitive than Cobas.
\end{abstract}

\section{Introduction}

Patients with advanced epidermal growth factor receptor (EGFR) mutated lung adenocarcinoma are treated with first- or second-generation EGFR tyrosine kinase inhibitors (TKIs). Approximately 50-75\% of these patients, however,

Martin Filipits

martin.filipits@meduniwien.ac.at

1 Institute of Cancer Research, Department of Medicine I, Comprehensive Cancer Center, Medical University of Vienna, Borschkegasse 8a, 1090 Vienna, Austria

2 Institute for Pathology and Bacteriology, Otto Wagner Hospital, Vienna, Austria

3 Department of Respiratory and Critical Care Medicine, and Ludwig Boltzmann Institute of COPD and Respiratory Epidemiology, Otto Wagner Hospital and Sigmund Freud University, Medical School, Vienna, Austria

4 Wilhelminenspital, Vienna, Austria

5 Division of Oncology, Department of Medicine I, Comprehensive Cancer Center, Medical University of Vienna, Vienna, Austria

\section{Key Points}

Analysis of cell-free DNA from blood for identifying genomic changes in the tumor of patients with advanced NSCLC is becoming more and more important.

Different technologies are available for EGFR mutation detection in cell-free plasma DNA from NSCLC patients.

Droplet digital PCR is more sensitive than Cobas ${ }^{\circledR}$ EGFR Mutation Test v2 for detecting EGFR T790M mutations in cell-free plasma DNA.

will develop the p.Thr790 Met (T790M) resistance mutation [1]. Osimertinib, a third-generation EGFR-TKI, inhibits activating-EGFR mutations and the T790M mutation but spares wild-type EGFR [2]. The clinical value of osimertinib was demonstrated in two phase III trials [3, 4]. In the AURA3 trial, osimertinib showed a PFS benefit over platinum-based chemotherapy in advanced EGFR-mutated non-small-cell 
lung cancer (NSCLC) patients who had developed T790Mmediated resistance under treatment with EGFR-TKIs [3]. Based on this trial, osimertinib has been approved for these patients. The presence of the T790M resistance mutation in tumor cells or cell-free plasma DNA has to be proven prior to initiation of second-line treatment with osimertinib. The FLAURA phase III trial demonstrated a PFS benefit for osimertinib compared to erlotinib or gefitinib in the first-line treatment of NSCLC patients with EGFR exon 19 deletions or L858R mutations independent of the T790M status [4]. Subsequently, osimertinib was approved as a first-line treatment of patients with metastatic NSCLC whose tumors have EGFR exon 19 deletions or L858R mutations, as detected by a US Food and Drug Administration (FDA)-approved test.

While initial studies analyzed $E G F R$ mutations in tumor biopsies, several studies suggested that the analysis of cellfree plasma DNA is a clinically useful alternative [5-8]. The analysis of cell-free plasma DNA from blood samples (liquid biopsy) supported by tumor tissue analyses in plasma-negative cases may now be the preferred strategy to select EGFRmutated NSCLC patients for osimertinib treatment [6-8]. At present, the semiquantitative PCR-based Cobas ${ }^{\circledR} E G F R$ Mutation Test v2 (Cobas) (Roche Molecular Systems, Pleasanton, CA, USA) is one of the FDA-approved plasma genotyping assays [9]. Highly sensitive genotyping assays, such as droplet digital polymerase chain reaction (ddPCR), can also reliably detect mutations in cell-free plasma DNA with high sensitivity and specificity [6-8]. The aim of our study was to compare ddPCR with Cobas with regard to their ability to detect three common EGFR mutations (T790M, exon 19 deletions, and L858R) in cell-free plasma DNA.

\section{Patients and Methods}

\subsection{Patients}

Patients with advanced EGFR-mutated lung adenocarcinoma were recruited at the Department of Respiratory and Critical Care Medicine, and Ludwig Boltzmann Institute of COPD and Respiratory Epidemiology, Otto Wagner Hospital, Vienna and Wilhelminenspital, Vienna between February 2016 and August 2017. All patients had histologically confirmed lung adenocarcinoma with EGFR mutations in their initial biopsy at diagnosis. Blood samples for plasma genotyping were collected within the scope of diagnostic routine procedures and were available in all patients. Plasma genotyping using ddPCR was performed at the Institute of Cancer Research, Department of Medicine I, Medical University of Vienna. The Cobas test was conducted at the Institute for Pathology and Bacteriology, Otto Wagner Hospital, Vienna. Sample collection and analysis was performed in accordance with the local ethics committee (EK No. 1132/2016).
All patients gave their written informed consent for providing blood specimens for plasma genotyping. Sixty-one patients had been included in a previous study on plasma DNA analysis for guiding osimertinib treatment [8].

\subsection{Plasma Genotyping}

Blood processing for plasma preparation and storage was performed as previously described [8]. Briefly, blood samples were collected in cell-free DNA blood collection tubes (BCTs) (Streck, La Vista, NE, USA) or cell-free DNA Blood Collection Tubes (Roche, Pleasanton, CA, USA). Two blood samples $(8 \mathrm{ml}$ each) were collected from all patients at each time point. One sample was analyzed with ddPCR (Bio-Rad Laboratories, Hercules, CA, USA) and the other one with Cobas (Roche Molecular Systems, Pleasanton, CA, USA).

To isolate plasma, whole blood was centrifuged at $200 \mathrm{~g}$ for $10 \mathrm{~min}$ and subsequently at $1600 \mathrm{~g}$ for $10 \mathrm{~min}$. Finally, the supernatant was collected and centrifuged at $1900 \mathrm{~g}$ for $10 \mathrm{~min}$.

For ddPCR, cell-free plasma DNA was extracted from $2 \mathrm{ml}$ plasma using the QIAamp circulating nucleic acid kit (Qiagen, Venlo, The Netherlands) according to the manufacturer's instructions. For the Cobas assay, extraction of cell-free plasma DNA was performed from $4 \mathrm{ml}$ of plasma using the Cobas ${ }^{\circledR}$ cfDNA Sample Preparation Kit according to the manufacturer's instructions.

T790M, exon 19 deletions, and L858R mutations were assessed by using the QX-200 ${ }^{\mathrm{TM}}$ ddPCR system (Bio-Rad, Hercules, CA, USA) and the Cobas z 480 Analyzer according to the manufacturer's instructions. The Cobas test determines several activating EGFR mutations (including exon 19 deletions and the L858R mutation) and the T790M resistance mutation simultaneously in one assay, whereas testing with ddPCR requires specific assays for each $E G F R$ mutation.

Primer and probes for ddPCR assays were custommade by Life Technologies (Carlsbad, CA, USA) and used as previously described [8]. Analysis of ddPCR data was performed with QuantaSoft analysis software (Bio-Rad). Results were reported as copies of mutant allele per ml of plasma. All ddPCR assays were analyzed in triplicate and results were reported as copies of mutant allele per $\mathrm{ml}$ of plasma. The threshold for positivity was $>1 \mathrm{copy} / \mathrm{ml}$ for T790M, exon 19 deletions, and L858R mutations.

\subsection{Statistical Analyses}

To calculate an estimate of agreement between ddPCR and Cobas, concordance rate (overall percent agreement [OPA]), sensitivity (positive percent agreement [PPA]), 
Table 1 Calculation of concordance rate, sensitivity, and specificity

\begin{tabular}{llll}
\hline & Cobas & & Total \\
\cline { 2 - 3 } & Negative & Positive & \\
\hline ddPCR & & & \\
Negative & $\mathrm{a}^{*}$ & $\mathrm{~b}^{\dagger}$ & $\mathrm{a}+\mathrm{b}$ \\
Positive & $\mathrm{c}^{\ddagger}$ & $\mathrm{d}^{\S}$ & $\mathrm{c}+\mathrm{d}$ \\
Total & $\mathrm{a}+\mathrm{c}$ & $\mathrm{b}+\mathrm{d}$ & $\mathrm{N}$ \\
\hline
\end{tabular}

$d d P C R$ droplet digital PCR, Cobas Cobas ${ }^{\circledR}$ EGFR Mutation Test v2

$* a$ the number of Cobas negative, ddPCR negative

${ }^{\dagger} b$ the number of Cobas positive, ddPCR negative

${ }^{\ddagger} c$ the number of Cobas negative, ddPCR positive

${ }^{\S} d$ the number of Cobas positive, ddPCR positive

and specificity (negative percent agreement [NPA]) were calculated from standard $2 \times 2$ tables as shown in Table 1 . The following formulas were used to calculate concordance rate, sensitivity, and specificity:

$$
\begin{aligned}
\text { Concordance rate } & =100 \% \times(a+d) /(a+b+c+d) \\
\text { Sensitivity } & =100 \% \times d /(b+d) \\
\text { Specificity } & =100 \% \times a /(a+c) .
\end{aligned}
$$

Comparison of the copy number ratio of T790M and activating mutations with response to osimertinib was done with the Kruskal-Wallis-Test.

All statistical data were analyzed with IBM SPSS Statistics software, version 23 (SPSS, IBM Corporation, Armonk, NY, USA).

\section{Results}

\subsection{Patient Characteristics}

In total, 354 plasma samples from 129 patients with advanced $E G F R$-mutated lung adenocarcinoma were analyzed simultaneously for T790M by both ddPCR and Cobas. The blood samples were collected at different time points and stages during the course of the disease. The number of longitudinal samples obtained from each patient varied between one and eight samples.

Characteristics of patients are summarized in Table 2. All patients had lung adenocarcinoma histology, stage IV disease, and had been treated with at least one EGFRTKI prior to collection of blood samples. Seventyseven T790M-positive patients $(60 \%)$ were treated with osimertinib.

Exon 19 deletions were determined simultaneously by both assays in 196 samples obtained from 71 patients with confirmed exon 19 deletion in tissue at the time of diagnosis. L858R mutation was assessed in 124 samples of 44 patients with confirmed L858R positive tissue genotype at diagnosis (including one L858R/S768I and one L858R/ Exon 18 double mutation). The samples of 14 patients with rare EGFR mutations (Table 2) were examined by ddPCR only for the presence of the T790M mutation.

\section{2 ddPCR}

T790M testing by ddPCR was performed in all 354 plasma samples collected from 129 patients (Table 3). T790M was detected in 53 of $129(41 \%)$ patients and in 71 of $354(20 \%)$ plasma samples (Table 3a). The T790M copy number in the 71 samples ranged from 1.5 to 6285 copies/ml (Fig. 1a).

Exon 19 deletions were detected in 40 of $71(56 \%)$ patients and 72 of 196 (37\%) samples (Table 3b). L858R

Table 2 Patient characteristics

\begin{tabular}{lc}
\hline & Patients $n(\%) *$ \\
\hline Total & $129(100 \%)$ \\
Age & \\
Median (range), y & $67(35-86)$ \\
$<65$ & $53(41 \%)$ \\
$\geq 65$ & $76(59 \%)$ \\
Sex & \\
Female & $102(79 \%)$ \\
Male & $27(21 \%)$ \\
Adenocarcinoma & $129(100 \%)$ \\
EGFR tissue genotype & \\
Exon 19 deletion & $71(55 \%)$ \\
L858R & $42(33 \%)$ \\
G719X & $5(4 \%)$ \\
L861Q & $4(3 \%)$ \\
Exon 20 insertion & $2(2 \%)$ \\
L858R/Exon 18 mutation & $1(0.8 \%)$ \\
L858R/S768I & $1(0.8 \%)$ \\
G719X/S768I & $1(0.8 \%)$ \\
L861Q/G719X & $1(0.8 \%)$ \\
S768I & $1(0.8 \%)$ \\
Previous EGFR-TKI therapy & \\
Afatinib & $74(57 \%)$ \\
Gefitinib & $36(28 \%)$ \\
Erlotinib & $3(2 \%)$ \\
No TKI & $14(11 \%)$ \\
Samples per patient & $2(2 \%)$ \\
Median (range) & \\
\hline & \\
&
\end{tabular}

TKI tyrosine kinase inhibitor

*Percentages may not total $100 \%$ because of rounding 
was found in 23 of 44 (52\%) patients and 42 of 124 (34\%) plasma samples (Table 3c). The lower frequency of EGFR mutations in these plasma samples compared to the literature and our own data may be explained by differences in treatment status between the present study and others. In our study, a substantial number of samples were obtained from patients who achieved a complete response during EGFR-TKI treatment.

\subsection{Cobas}

Using the Cobas assay, T790M was detected in 17 of $129(13 \%)$ patients and 20 of $354(6 \%)$ plasma samples (Table 3a). Exon 19 deletions were detected in 36 of 71 (51\%) patients and 63 of 196 (32\%) samples (Table 3b). L858R was found in 21 of $44(47 \%)$ patients and 35 of $124(28 \%)$ plasma samples (Table 3c).

\subsection{Comparison Between ddPCR and Cobas}

Contingency tables for each EGFR mutation detected by ddPCR or Cobas are presented in Table $3 \mathrm{a}-\mathrm{c}$. The concordance rate, sensitivity, and specificity between ddPCR and Cobas was dependent on the type of EGFR mutation.

With regard to T790M, 51 of 354 (14\%) plasma samples were positive with ddPCR but negative with Cobas (Table 3a). T790M positivity in ddPCR-positive but Cobasnegative plasma samples ranged from 1.5 to 52 copies $/ \mathrm{ml}$.
Except for two cases, the mutant copy numbers in the 51 discordant samples (ddPCR positive/Cobas negative) were below 25 copies $/ \mathrm{ml}$, which is the detection limit of Cobas. All samples that were T790M positive with Cobas were also positive with ddPCR (Fig. 1a). These results led to a concordance rate of $86 \%$, a sensitivity of $100 \%$, and a specificity of $85 \%$ (Table $3 \mathrm{a}$ ).

Among 196 samples tested for exon 19 deletions with both platforms, 14 samples were ddPCR positive/Cobas negative (exon 19 deletion-positivity ranged from 2 to 136 copies/ml) and five samples were ddPCR negative/Cobas positive (Table $3 b$ ). With regard to exon 19 deletions, the comparison of ddPCR and Cobas resulted in a concordance rate of $90 \%$, a sensitivity of $92 \%$, and a specificity of $89 \%$.

In 124 samples, the L858R mutation was analyzed by both platforms. Comparison revealed 14 discordant results including ten samples that were ddPCR positive/Cobas negative (L858R-positivity ranged from 1.3 to 226 copies $/ \mathrm{ml}$ ) and three samples that were ddPCR negative/Cobas positive (Table 3c). With regard to L858R, comparison of ddPCR and Cobas revealed a concordance rate of $90 \%$, a sensitivity of $91 \%$, and a specificity of $89 \%$.

\subsection{Comparison Between ddPCR and Cobas in Patients Who Progressed under Treatment with an EGFR TKI}

At the time of disease progression to first- or second generation EGFR-TKI therapy, 50 out of 129 (39\%) patients

Table 3 Contingency tables comparing test results of ddPCR and Cobas for EGFR T790M, exon 19 deletions, and L858R in cell-free plasma DNA

\begin{tabular}{|c|c|c|c|c|}
\hline & \multicolumn{2}{|l|}{ Cobas } & \multirow[t]{2}{*}{ Total } & \\
\hline & Negative & Positive & & \\
\hline \multicolumn{5}{|l|}{ (a) $\mathrm{T} 790 \mathrm{M}$} \\
\hline \multicolumn{5}{|l|}{ ddPCR } \\
\hline Negative & $283(79.9 \%)$ & $0(0.0 \%)$ & $283(79.9 \%)$ & Concordance rate $86 \%$ \\
\hline Positive & $51(14.4 \%)$ & $20(5.6 \%)$ & $71(20.1 \%)$ & Sensitivity $100 \%$ \\
\hline Total & $334(94.4 \%)$ & $20(5.6 \%)$ & $354(100 \%)$ & Specificity $85 \%$ \\
\hline \multirow{2}{*}{\multicolumn{5}{|c|}{$\begin{array}{l}\text { (b) Exon } 19 \text { deletions } \\
\text { ddPCR }\end{array}$}} \\
\hline & & & & \\
\hline Negative & $119(60.7 \%)$ & $5(2.6 \%)$ & $124(63.3 \%)$ & Concordance rate $90 \%$ \\
\hline Positive & $14(7.1 \%)$ & $58(29.6 \%)$ & $72(36.7 \%)$ & Sensitivity $92 \%$ \\
\hline Total & $133(67.9 \%)$ & $63(32.1 \%)$ & $196(100 \%)$ & Specificity $89 \%$ \\
\hline \multicolumn{5}{|l|}{ (c) L858R } \\
\hline \multicolumn{5}{|l|}{ ddPCR } \\
\hline Negative & $79(63.7 \%)$ & $3(2.4 \%)$ & $82(66.1 \%)$ & Concordance rate $90 \%$ \\
\hline Positive & $10(8.1 \%)$ & $32(25.8 \%)$ & $42(33.9 \%)$ & Sensitivity $91 \%$ \\
\hline Total & $89(71.8 \%)$ & $35(28.2 \%)$ & $124(100 \%)$ & Specificity $89 \%$ \\
\hline
\end{tabular}

Values are given as number (percent)

$d d P C R$ droplet digital PCR, Cobas Cobas ${ }^{\circledR}$ EGFR Mutation Test v2 
a
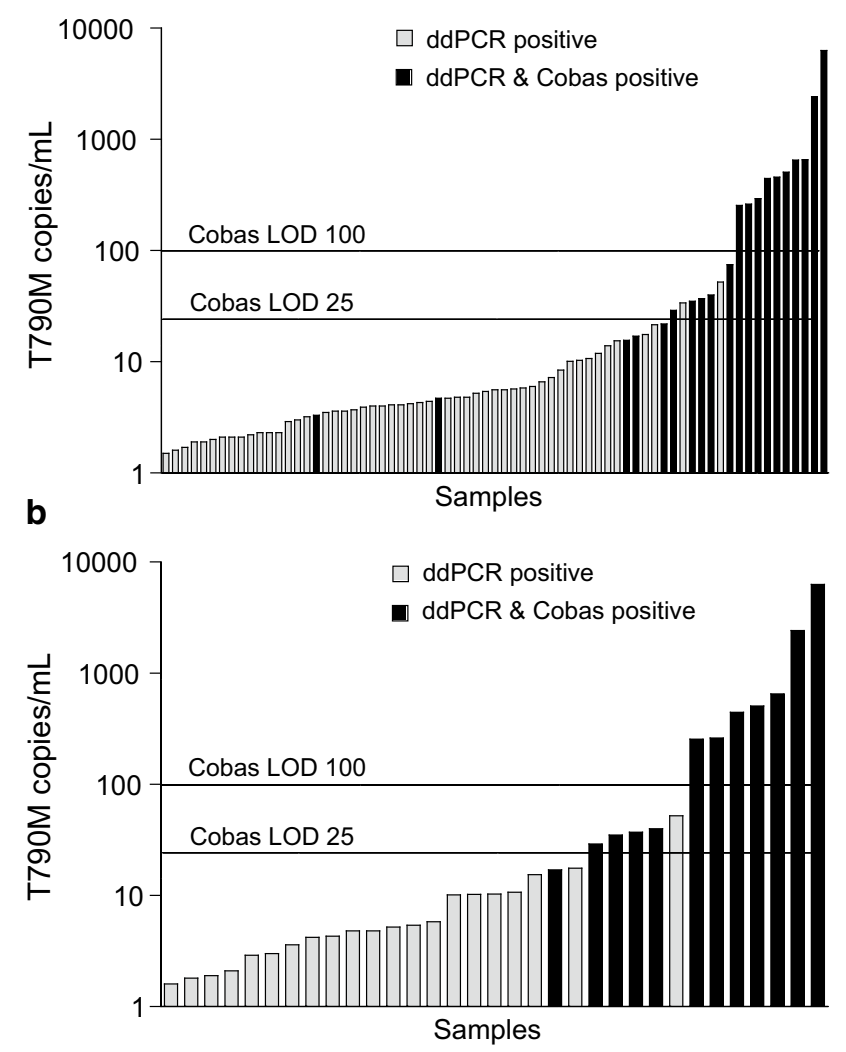

Fig. 1 T790M copy numbers of ddPCR-positive/Cobas-negative and ddPCR-positive/Cobas-positive cases in all 71 T790M-positive samples (a) and in 33 T790M-positive patients who progressed under treatment with a first- or second-generation EGFR-TKI (b). Twenty of $71(28 \%)$ samples (a) and 12 of $33(36 \%)$ samples (b) were T790M positive in both ddPCR and Cobas analysis, respectively. The Cobas limit of detection (LOD) is $25 \mathrm{~T} 790 \mathrm{M}$ copies/ml for intact DNA and $100 \mathrm{~T} 790 \mathrm{M}$ copies/ml for fragmented DNA (200 bp)

were analyzed for T790M in the plasma. In the remaining $79(61 \%)$ patients, the feasibility of treatment monitoring was investigated.

In patients who had progressive disease, 33 of 50 (66\%) were T790M positive using ddPCR and the T790M copy number ranged from 1.6 to $6285 \mathrm{copies} / \mathrm{ml}$ (Fig. 1b). The copy number ratio of $\mathrm{T} 790 \mathrm{M}$ and activating mutations ranged from 0.01 to 6.85 . In ten patients only T790M but not the corresponding activating mutation was detected (six patients with exon 19 deletions and four patients with L858R). In patients with progressive disease who were tested with Cobas, 12 of 50 (24\%) were T790M positive and the corresponding semi-quantitative index (SQI) values ranged from 5.0 to 13.61 . The $\mathrm{T} 790 \mathrm{M}$ positivity rate in patients who were tested for the purpose of treatment monitoring was $27 \%$ (21/79) using ddPCR, but only 6\% (5/79) using Cobas.

In 20 of 50 (40\%) patients with disease progression an additional tissue re-biopsy was available and T790M was detected in six out of the $20(30 \%)$ cases. All of the six patients who were T790M positive in tissue analyses were also positive in plasma using ddPCR for mutation detection but negative when the Cobas test was used, whereas in the ten patients who were T790M negative in the tissue re-biopsy, five were T790M positive using ddPCR and three were T790M positive using Cobas (these patients were also T790M positive with ddPCR). In four patients the tissue rebiopsy was not evaluable.

Thirty-two of the 33 T790M-positive patients who were tested with ddPCR and who progressed under treatment with a first- or second-generation EGFR-TKI received osimertinib. The response rate in 28 of 32 patients who were evaluable for response was $61 \%$ (Table 4). The response rate was $44 \%$ in nine patients who were $\mathrm{T} 790 \mathrm{M}$ positive in both ddPCR and Cobas analysis and $68 \%$ in 19 patients who were T790M positive with ddPCR but negative with Cobas (Table 4). The copy number ratio of T790M and activating mutations was not significantly associated with response to osimertinib. Patients who responded to osimertinib therapy had a median copy number ratio of 0.27 compared to 0.08 in patients who did not respond to osimertinib $(p=0.2)$.

\section{Discussion}

In the present study, we compared the performance of ddPCR and Cobas for detecting T790M, exon 19 deletions, and L858R mutations in plasma samples from patients with advanced $E G F R$-mutated lung adenocarcinoma. We demonstrated that ddPCR detected a substantially higher number of T790M mutations in plasma samples compared to Cobas, whereas differences between ddPCR and Cobas were smaller in case of exon 19 deletions and L858R. We and others have previously shown that ddPCR is highly sensitive, allows absolute quantification of mutations, and is clinically useful for the detection of EGFR mutations in cell-free plasma DNA and clinically relevant for selecting patients for osimertinib treatment [6-8]. The biggest advantage of ddPCR is its high sensitivity and specificity, while a major disadvantage is its limited multiplexing capacity. The Cobas ${ }^{\circledR}$ EGFR Mutation Test v2 is a real-time PCR test for the qualitative detection of 42 mutations in the EGFR gene [9]. It has been approved by the FDA as companion diagnostic to erlotinib and osimertinib for the detection of T790M, exon 19 deletions, and L858R mutations in tissue and plasma. The clinical utility of the Cobas ${ }^{\circledR}$ EGFR Mutation Test $\mathrm{v} 2$ for T790M detection has been previously shown in cell-free plasma DNA samples obtained from patients who had been enrolled in the AURA3 phase III trial [3].

A cross-platform comparison of different technologies (cobas ${ }^{\circledR} E G F R$ Mutation Test, Therascreen ${ }^{\mathrm{TM}}$ EGFR ARMS-PCR, ddPCR, BEAMing dPCR) for EGFR mutation 
Table 4 Response to osimertinib in evaluable patients who were T790M positive with ddPCR

\begin{tabular}{lclc}
\hline & All patients $(n=28)$ & Cobas T790M positive $(n=9)$ & Cobas T790M negative $(n=19)$ \\
\hline Response & & & \\
Complete response & $3(10.7 \%)$ & $0(0 \%)$ & $3(15.7 \%)$ \\
Partial response & $14(50.0 \%)$ & $4(44.4 \%)$ & $10(52.6 \%)$ \\
Stable disease & $4(14.3 \%)$ & $2(22.2 \%)$ & $2(10.5 \%)$ \\
Disease progression & $7(25.0 \%)$ & $3(33.3 \%)$ & $4(21.1 \%)$ \\
\hline
\end{tabular}

Values are given as number (\%)

Percentages may not total 100 because of rounding

detection in cell-free plasma DNA from NSCLC patients revealed that, at least for the T790M mutation, the digital platforms outperformed the non-digital platforms [10], which is in line with our findings. In the study by Thress et al., EGFR mutation status was analyzed with the four different plasma assays in 38 plasma samples from the AURA trial and the results were compared with tissue test results [10]. Similar to our results, this comparison showed that ddPCR has a higher sensitivity in detecting the T790M mutation than Cobas. Furthermore, cobas ${ }^{\circledR} E G F R$ Mutation Test and BEAMing dPCR were performed in 72 additional plasma samples. The two platforms demonstrated high sensitivity (82-87\%) and specificity (97\%) for EGFR-sensitizing mutations. For the T790M mutation, BEAMing dPCR had a higher sensitivity than the cobas ${ }^{\circledR} E G F R$ Mutation Test ( $81 \%$ vs. $73 \%$ ). However, the concordance between the two platforms was $90 \%$ for T790M, $90 \%$ for exon 19 deletions, and $93 \%$ for L858R. In our larger study, we observed a similarly high concordance between ddPCR and Cobas $-86 \%$ for T790M, 90\% for exon 19 deletions, and $89 \%$ for L858R. Nevertheless, ddPCR is more sensitive than Cobas in detecting T790M, exon 19 deletions, and L858R mutations.

Differences in mutation detection between Cobas and ddPCR were most evident for T790M testing. Fifty-one samples were T790M positive if assessed with ddPCR but T790M negative if tested with Cobas. Interestingly, 49 of these samples had a copy number below 25 copies/ml and 41 had a copy number below 10 copies $/ \mathrm{ml}$. The relatively high limit of detection of Cobas for the T790M mutation (25 copies $/ \mathrm{ml}$ for intact DNA and 100 copies $/ \mathrm{ml}$ for truncated DNA fragments of $200 \mathrm{bp}$ ) may explain the smaller number of T790M-positive samples detected using this method.

Our results are clinically meaningful because the T790M positivity rate of the 50 patients who progressed under treatment with an EGFR TKI was $66 \%$ in the present study, which is in line with previous reports from us and others [8, 11-13]. In addition, all six patients who were T790Mpositive in tissue analyses were also positive in plasma using ddPCR for mutation detection but negative when the Cobas test was used. Finally, the response rate in 28 evaluable patients who received osimertinib was $61 \%$, which is also similar to previous findings $[3,8]$. The detection of low T790M copy numbers $(<10$ copies/ml $)$ may be clinically relevant because we have previously shown that these patients have a similar response to osimertinib compared to patients with a higher T790M copy number ( $>10$ copies $/ \mathrm{ml}$ ) [8]. Thus, in an important fraction of patients with advanced EGFR-mutated NSCLC who had progressed under treatment with an EGFR-TKI and who would benefit from osimertinib, T790M is not detected by Cobas.

In summary, we observed a high concordance between ddPCR and Cobas in detecting EGFR mutations in plasma samples of patients with advanced EGFR-mutated lung adenocarcinoma but ddPCR was more sensitive than Cobas.

Acknowledgements Open access funding provided by Medical University of Vienna.

\section{Compliance with Ethical Standards}

Funding The study was funded by AstraZeneca. Open access funding provided by Medical University of Vienna.

Conflict of interest Anna Buder has received honoraria from AstraZeneca. Maximilian J. Hochmair has received honoraria from AstraZeneca, Bristol-Myers Squibb, Boehringer Ingelheim, Merck Sharp \& Dohme, Pfizer, and Roche and had consulting or advisory roles with Boehringer Ingelheim, Merck Sharp \& Dohme, Novartis, and Roche. Klaus Kirchbacher received honoraria from AstraZeneca, Boehringer Ingelheim, Pfizer, and Roche. Otto C. Burghuber received honoraria from AstraZeneca, Boehringer Ingelheim, Glaxo Smith Kline, Menarini, Chiesi, Takeda, Roche, and Novartis. Robert Pirker has received honoraria from AstraZeneca, Boehringer Ingelheim, and Clovis. Martin Filipits has received honoraria from Astra Zeneca, Boehringer Ingelheim, Eli Lilly, Merck Sharp \& Dohme, Novartis, Ratiopharm, and Roche. Ulrike Setinek, Sophia Schwab, and Andrea Keck declare that they have no conflicts of interest that might be relevant to the contents of this manuscript.

Open Access This article is distributed under the terms of the Creative Commons Attribution-NonCommercial 4.0 International License (http://creativecommons.org/licenses/by-nc/4.0/), which permits any noncommercial use, distribution, and reproduction in any medium, provided you give appropriate credit to the original author(s) and the source, provide a link to the Creative Commons license, and indicate if changes were made. 


\section{References}

1. Pao W, Miller VA, Politi KA, Riely GJ, Somwar R, Zakowski MF, et al. Acquired resistance of lung adenocarcinomas to gefitinib or erlotinib is associated with a second mutation in the EGFR kinase domain. PLoS Med. 2005;2(3):e73. https://doi.org/10.1371/journ al.pmed.0020073.

2. Cross DA, Ashton SE, Ghiorghiu S, Eberlein C, Nebhan CA, Spitzler PJ, et al. AZD9291, an irreversible EGFR TKI, overcomes T790M-mediated resistance to EGFR inhibitors in lung cancer. Cancer Discov. 2014;4(9):1046-61. https://doi.org/10.1158/21598290.CD-14-0337.

3. Mok TS, Wu YL, Ahn MJ, Garassino MC, Kim HR, Ramalingam SS, et al. Osimertinib or platinum-pemetrexed in EGFR T790Mpositive lung cancer. N Engl J Med. 2017;376(7):629-40. https:// doi.org/10.1056/NEJMoa1612674.

4. Soria JC, Ohe Y, Vansteenkiste J, Reungwetwattana T, Chewaskulyong $\mathrm{B}$, Lee $\mathrm{KH}$, et al. Osimertinib in untreated EGFRmutated advanced non-small-cell lung cancer. N Engl J Med. 2018;378(2):113-25. https://doi.org/10.1056/NEJMoa1713137.

5. Buder A, Tomuta C, Filipits M. The potential of liquid biopsies. Curr Opin Oncol. 2016;28(2):130-4. https://doi.org/10.1097/ CCO.0000000000000267.

6. Oxnard GR, Paweletz CP, Kuang Y, Mach SL, O'Connell A, Messineo MM, et al. Noninvasive detection of response and resistance in EGFR-mutant lung cancer using quantitative nextgeneration genotyping of cell-free plasma DNA. Clin Cancer Res. 2014;20(6):1698-705. https://doi.org/10.1158/1078-0432. CCR-13-2482.

7. Sacher AG, Paweletz C, Dahlberg SE, Alden RS, O'Connell A, Feeney N, et al. Prospective validation of rapid plasma genotyping for the detection of EGFR and KRAS mutations in advanced lung cancer. JAMA Oncol. 2016;2(8):1014-22. https://doi.org/10.1001/ jamaoncol.2016.0173.

8. Buder A, Hochmair MJ, Schwab S, Bundalo T, Schenk P, Errhalt $\mathrm{P}$, et al. Cell-free plasma DNA-guided treatment with osimertinib in patients with advanced EGFR-mutated NSCLC. J Thorac Oncol. 2018;13(6):821-30. https://doi.org/10.1016/j. jtho.2018.02.014.

9. Brown P. The Cobas(R) EGFR Mutation Test v2 assay. Future Oncol. 2016;12(4):451-2. https://doi.org/10.2217/fon.15.311.

10. Thress KS, Brant R, Carr TH, Dearden S, Jenkins S, Brown $\mathrm{H}$, et al. EGFR mutation detection in ctDNA from NSCLC patient plasma: a cross-platform comparison of leading technologies to support the clinical development of AZD9291. Lung Cancer. 2015;90(3):509-15. https://doi.org/10.1016/j.lungc an.2015.10.004

11. Reckamp KL, Melnikova VO, Karlovich C, Sequist LV, Camidge $\mathrm{DR}$, Wakelee $\mathrm{H}$, et al. A highly sensitive and quantitative test platform for detection of NSCLC EGFR mutations in urine and plasma. J Thorac Oncol. 2016;11(10):1690-700. https://doi. org/10.1016/j.jtho.2016.05.035.

12. Sundaresan TK, Sequist LV, Heymach JV, Riely GJ, Janne PA, Koch WH, et al. Detection of T790M, the acquired resistance EGFR mutation, by tumor biopsy versus noninvasive bloodbased analyses. Clin Cancer Res. 2016;22(5):1103-10. https:// doi.org/10.1158/1078-0432.CCR-15-1031.

13. Hochmair MJ, Buder A, Schwab S, Burghuber OC, Prosch H, Hilbe W, et al. Liquid-biopsy-based identification of EGFR T790M mutation-mediated resistance to afatinib treatment in patients with advanced EGFR mutation-positive NSCLC, and subsequent response to osimertinib. Target Oncol. 2018. https:// doi.org/10.1007/s11523-018-0612-z. 